\title{
Computer-aided differential diagnosis system for Alzheimer's disease based on machine learning with functional and morphological image features in magnetic resonance imaging
}

\author{
Yasuo Yamashita ${ }^{1,2}$, Hidetaka Arimura ${ }^{3 *}$, Takashi Yoshiura ${ }^{4}$, Chiaki Tokunaga ${ }^{2}$, Ohara Tomoyuki ${ }^{5}$, \\ Koji Kobayashi $^{2}$, Yasuhiko Nakamura ${ }^{2}$, Nobuyoshi Ohya ${ }^{2}$, Hiroshi Honda ${ }^{4}$, Fukai Toyofuku ${ }^{3}$ \\ ${ }^{1}$ Graduate School of Medical Science, Kyushu University, Fukuoka, Japan \\ ${ }^{2}$ Division of Radiology, Department of Medical Technology, Kyusyu University Hospital, Fukuoka, Japan \\ ${ }^{3}$ Faculty of Medical Science, Kyushu University, Fukuoka, Japan \\ ${ }^{4}$ Department of Clinical Radiology, Graduate School of Medical Sciences, Kyushu University, Fukuoka, Japan \\ ${ }^{5}$ Department of Neuropsychiatry, Graduate School of Medical Sciences, Kyushu University, Fukuoka, Japan \\ Email: ${ }^{*}$ arimurah@med.kyushu-u.ac.jp \\ Received 1 September 2013; revised 8 October 2013; accepted 25 October 2013 \\ Copyright (C) 2013 Yasuo Yamashita et al. This is an open access article distributed under the Creative Commons Attribution License, \\ which permits unrestricted use, distribution, and reproduction in any medium, provided the original work is properly cited.
}

\begin{abstract}
Alzheimer's disease (AD) is a dementing disorder and one of the major public health problems in countries with greater longevity. The cerebral cortical thickness and cerebral blood flow (CBF), which are considered as morphological and functional image features, respectively, could be decreased in specific cerebral regions of patients with dementia of Alzheimer type. Therefore, the aim of this study was to develop a computer-aided classification system for AD patients based on machine learning with the morphological and functional image features derived from a magnetic resonance (MR) imaging system. The cortical thicknesses in ten cerebral regions were derived as morphological features by using gradient vector trajectories in fuzzy membership images. Functional CBF maps were measured with an arterial spin labeling technique, and ten regional CBF values were obtained by registration between the CBF map and Talairach atlas using an affine transformation and a free form deformation. We applied two systems based on an arterial neural network (ANN) and a support vector machine (SVM), which were trained with 4 morphological and 6 functional image features, to 15 AD patients and 15 clinically normal (CN) subjects for classification of $\mathrm{AD}$. The area under the receiver operating characteristic curve (AUC) values for the two systems based on the ANN and SVM with both image
\end{abstract}

"Corresponding author. features were 0.901 and 0.915 , respectively. The AUC values for the ANN- and SVM-based systems with the morphological features were 0.710 and 0.660 , respectively, and those with the functional features were 0.878 and 0.903 , respectively. Our preliminary results suggest that the proposed method may have potential for assisting radiologists in the differential diagnosis of $\mathrm{AD}$ patients by using morphological and functional image features.

Keywords: Computer-aided Classification (CAD); Alzheimer's Disease; Magnetic Resonance Imaging (MRI); Arterial Spin Labeling (ASL); Fuzzy Membership Image; Cortical Thickness; Cerebral Blood Flow (CBF)

\section{INTRODUCTION}

Alzheimer's disease (AD) is the most common cause of dementia in the majority of developed countries [1-5]. $\mathrm{AD}$ is associated with morphological and functional changes, i.e., the atrophy of gray matter in the cerebral cortex, and the decrease of cerebral blood flow (CBF) in specific cerebral regions which can be evaluated with magnetic resonance imaging (MRI) and nuclear medicine examinations obtained by positron-emission tomography (PET) or single-photon emission computed tomography (SPECT) [6-13]. However, the examinations by PET and SPECT are more expensive and invasive than those using MRI. On the other hand, arterial spin labe- 
ling (ASL) is a cheaper and non-invasive MR imaging technique for the measurement of CBF without using contrast medium $[14,15]$. Yoshiura et al. suggested that the CBF map images measured by the ASL technique can be used to assist radiologists in the discrimination of patients with $\mathrm{AD}[16]$.

In recent years, various kinds of computer-aided diagnosis (CAD) methods for $\mathrm{AD}$ patients have been developed [17-21]. However, to the best of our knowledge, there is no CAD system for the classification of AD patients using machine learning with morphological and functional image features obtained by MR imaging alone. Therefore, our purpose in this study was to develop a computer-aided differential diagnosis system for $\mathrm{AD}$ patients based on machine learning with morphological and functional image features obtained by MR imaging without contrast medium.

\section{MATERIALS AND METHODS}

\subsection{Subjects and MR Data}

This study was approved by an institutional review board of the Kyushu University Hospital. We applied our proposed method to three-dimensional (3D) T1-weighted MR images of the whole brain and ASL images obtained from 30 cases, including 15 patients who were clinically diagnosed with $\mathrm{AD}$ by a neuropsychiatrist at Kyushu University Hospital (age range: 54 - 89 years; mean age: 77 years; Mini-Mental State Examination (MMSE) score: 11 - 25; mean: 22) and 15 cognitively normal (CN) subjects (age range: 68 - 86 years; mean age: 73 years; MMSE score: 28 - 30; mean: 29). These data were acquired on a 3.0-T MRI scanner (Intera Achieva 3.0 T Quasar Dual R2.1; PHILIPS Electronics, Best, Netherlands).

T1-weighted sequencing was performed using a magnetization prepared rapid gradient echo (MPRAGE) sequence (time of repetition (TR): $8.3 \mathrm{~ms}$; time of echo (TE): $3.8 \mathrm{~ms}$; time of inversion (TI): $240 \mathrm{~ms}$; flip angle: 8 degrees; sensitivity encoding (SENSE) factor: 2 ; number of samples averaged (NAS): $1 ; 240 \times 240 \times 150$ voxels; individual voxel size: $1.0 \mathrm{~mm} \times 1.0 \mathrm{~mm} \times 1.0$ $\mathrm{mm})$. ASL was performed using quantitative signal targeting by alternating radiofrequency pulses labeling of arterial regions (QUASAR), a pulsed ASL technique developed by Petersen et al. [22]. The QUASAR protocol consisted of two-dimensional image sequencing (labeling slab thickness: $150 \mathrm{~mm}$; gap between the labeling and imaging slabs: $15 \mathrm{~mm}$; SENSE factor: 2.5; TR: $4000 \mathrm{~ms}$; TE: $22 \mathrm{~ms}$; sampling interval: $300 \mathrm{~ms}$; sampling time points: 13 ; $64 \times 64$ matrix; individualvoxel size: $3.6 \mathrm{~mm}$ $\times 3.6 \mathrm{~mm} ; 84$ dynamics; seven transverse slices of 6.0 $\mathrm{mm}$ thickness (gap: $2 \mathrm{~mm}$ )). T2-weighted images (TR: $3000 \mathrm{~ms}$; TE: $105 \mathrm{~ms} ; 512 \times 512$ matrix; seven transverse slices of $6.0 \mathrm{~mm}$ thickness) were obtained at the same slice level as the ASL sequence.

\subsection{Proposed Method}

Our proposed method consists of three steps, i.e., the measurement of the functional and morphological image features, and the classification of AD patients based on machine learning. Figure 1 shows the overall scheme for the calculation of $\mathrm{AD}$ patients and $\mathrm{CN}$ subjects based on the functional and morphological image features. The average CBFs in 16 cerebral cortical regions were determined as functional image features based on the CBF map images obtained by the ASL technique. The average thicknesses in ten cerebral cortical regions were measured as morphological image features in 3D T1-weighted whole brain images. In the next step, a combination of functional and morphological image features for classification of $\mathrm{AD}$ patients was selected based on the statistical p-values and post studies in 16 average CBFs and ten cerebral thicknesses. Finally, AD patients and $\mathrm{CN}$ subjects were classified by using a machine learning technique, i.e., an arterial neural network (ANN) or a support vector machine (SVM).

\subsubsection{Measurement of Functional Image Features}

Average CBFs in 16 cerebral cortical regions were determined as functional image features based on the CBF map image, which was non-linearly aligned with the Talairach brain atlas by using a registration method with an affine transformation and a free form deformation (FFD). The Talairach brain atlas is one of the standard models labeled for functional human brain mapping, and consists

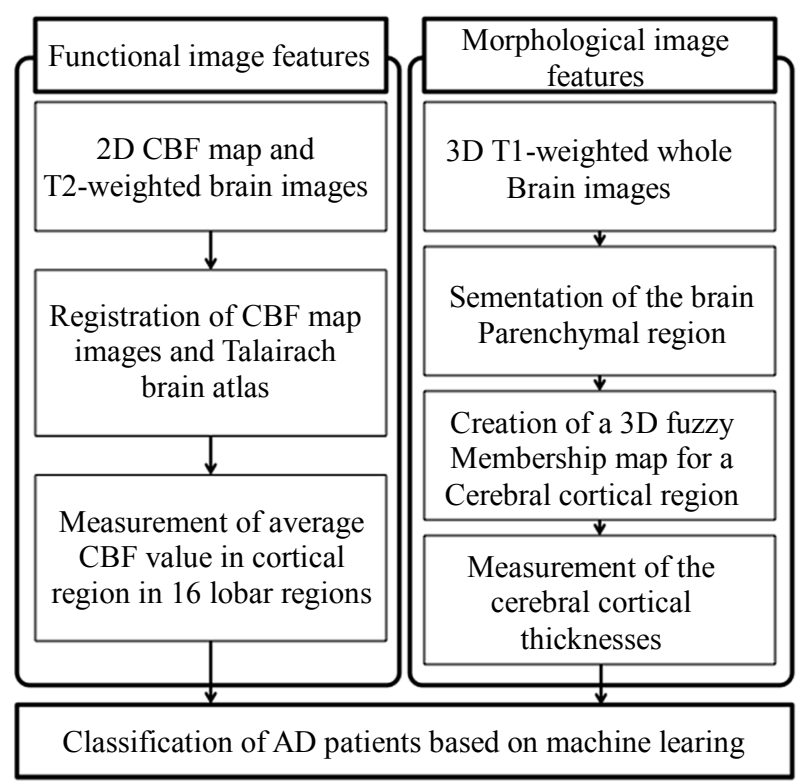

Figure 1. Overall scheme for the calculation of $\mathrm{AD}$ patients and $\mathrm{CN}$ subjects based on the functional and the morphological image features. 
of 5 levels (hemisphere, lobe, gyrus, tissue and cell level) [23-25]. The lobe and tissue levels were used for measuring the average $\mathrm{CBF}$ in each lobe of the cerebral cortical region.

We developed the registration method between the Talairach brain atlas and a CBF map image of a patient through the corresponding T2-weighted brain image. Figure 2 illustrates the registration procedure for measurement of the average CBF values in 16 cortical regions. Our registration method was composed of two steps. In the first step, an affine transformation was applied as a global registration. The affine transformation is given by

$$
\left(\begin{array}{c}
X_{n} \\
Y_{n} \\
1
\end{array}\right)=\left(\begin{array}{ccc}
C_{11} & C_{12} & C_{13} \\
C_{21} & C_{22} & C_{23} \\
0 & 0 & 1
\end{array}\right)\left(\begin{array}{c}
x_{n} \\
y_{n} \\
1
\end{array}\right)
$$

where $x_{n}$ and $y_{n}$ are the coordinates in the moving image (the CBF map image or the Talairach brain atlas), and $X_{n}$ and $Y_{n}$ are the coordinates in the deformed image. The affine transformation matrix consisting of $C_{11}$ to $C_{32}$ was obtained by using a least squaresmethod based on a singular value decomposition so that the feature points in the moving and reference images corresponded with each other. For determination of the affine transformation, the minimum and maximum coordinates of the binary images of the moving images and T2-weighted images were selected as four sets of corresponding feature points.

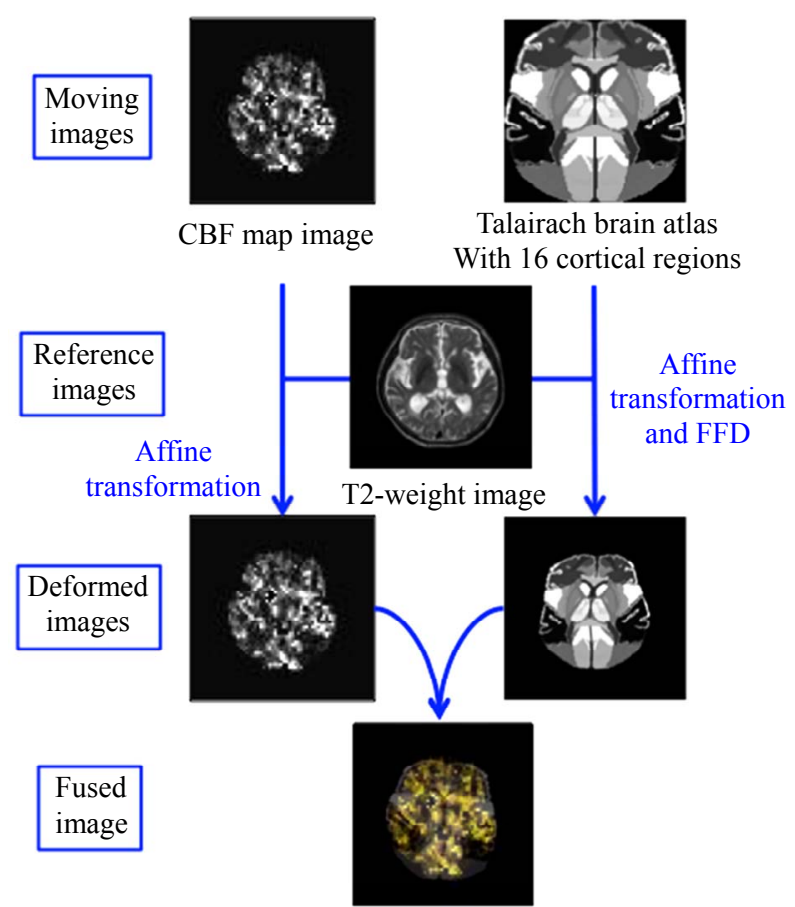

Figure 2. Flowchart of registration for extraction the average $\mathrm{CBF}$ values in 16 cortical regions.
In the second step, FFD was employed as a non-linear local registration [26] to register the Talairach brain atlas with the T2-weightedimages by determining the transformation function based on B-spline functions, from which a moving vector $\left(D_{x}, D_{y}\right)$ in a two-dimensional image was obtained. The moved coordinates $(X, Y)$ in the coordinate system in the T2-weighted image weredefined by

$$
(X, Y)=\left(x+D_{x, y}+D_{y}\right)
$$

where $x$ and $y$ are the original coordinates in the Talairach brain atlas. Sixty-four sets of corresponding feature points were determined by using a templatematching technique between the Talairach atlas and 64 subimages (matrix size: $128 \times 128$ ) obtained from the T2-weighted image. Each feature point was determined as the coordinates where the centers of the template subimage took the maximum cross-correltion coefficient in the Talairach atlas.

To approximate the moving distance space $D_{x, y}$, we formulate an approximation function $D_{x, y}$ as uniform bicubic B-spline functions, which were defined by using a control lattice $\Phi$ overlaid on the domain $\Omega$. We assumed that $\Phi$ is an $(m+3)(n+3)$ lattice which spans the integer grid in the domain $\Omega$. Let $\Phi_{i j}$ be the value of the control point on lattice $\Phi$, located at $(i, j)$ for $i=\mid 1,0 \cdots m+1$ and $j=\mid 1,0 \cdots n+1$. The approximation function $D(x, y)$ in the moving distance space was defined in terms of these control points by

$$
D_{x}(x, y)=\sum_{k 0}^{3} \sum_{l=0}^{3} B_{k}(s) B_{l}(t) \phi_{(i+k)(j+i)}
$$

where $i=\lfloor x\rfloor|1, \quad j=\lfloor y\rfloor| 1, s=x\rfloor x\rfloor$, $=y-y(0 \leq t<1), k=0,1,2,3$, and $l=0,1,2,3$. The functions $B_{k}$ and $B_{l}$ are uniform cubic B-spline basis functions defined as

$$
\begin{gathered}
B_{0}(t)=\frac{(1-t)^{3}}{6} \\
B_{1}(t)=\frac{3 t^{3}-6 t^{2}+4}{6} \\
B_{2}(t)=\frac{-3 t^{3}+3 t^{2}+3 t+1}{6} \\
B_{3}(t)=\frac{t^{3}}{6}
\end{gathered}
$$

We chose 16 cerebral cortical regions in the Talairach brain atlas, i.e., frontal, limbic, occipital, parietal, sublobar, temporal lobes, posterior cingulate gyri and precuneuses in the left and right brain hemispheres after the registration, where the average CBFs were measured. Our method applied cerebral cortical thicknesses as mor- 
phological image features for the classification of $\mathrm{AD}$ patients. Tokunaga et al. developed an automated method for measuring the 3D cerebral cortical thicknesses in $\mathrm{AD}$ patients based on 3D fuzzy membership maps derived from T1-weighted images, which includes the atrophy in the cortical and white matter regions determined on each cortical surface voxel by using membership profiles on trajectories of local gradient vectors in a fuzzy membership map [21]. For measurement of the cortical thicknesses in ten cerebral regions, we adopted Tokunaga's method. This method consisted of mainly three steps as follows:

1) Segmentation of the brain parenchymal region based on a brain model matching between a brain mask and a 3D T1-weighted image;

2) Creation of a fuzzy membership map for the cerebral cortical region based on the fuzzy c-means (FCM) clustering algorithm;

3) Calculation of the cerebral cortical thickness using localized gradient vector trajectories in fuzzy membership maps.

In order to investigate the regional atrophy at the lobe level, i.e., frontal, temporal, parietal, occipital lobes and insula for the left and right brain hemisphere, the cerebral cortical thicknesses were separately evaluated in ten lobar regions. The ten lobar regions were obtained by registration of the lobar model image to each brain parenchymal image by using the affine transformation and FFD. The lobar model image was selected from a probabilistic reference system for the human brain at the International Consortium for Brain Mapping (ICBM) website of the Laboratory of Neuro Imaging (LONI) [27].

\subsubsection{Classification of AD Patients}

We applied two machine learning classifiers, i.e., an ANN and a SVM, which were trained with the functional and morphological image features, to $15 \mathrm{AD}$ patients and $15 \mathrm{CN}$ subjects for classification of $\mathrm{AD}$. The in putfunctional features for the classifiers were the average CBF values in the six regions, i.e., the four lobes (left occipital lobe, left posterior cingulate gyrus, left and right precunei) where AD-related hypoperfusion was found in the previous step, and the two regions (right occipital lobe and right parietal lobe) where the hypoperfusion was expected based on previous reports [28]. In addition, the input morphological features were the average values of the cortical region thicknesses in four regions, i.e., the left and right temporal lobes, and the left and right insula, all of which showed statistically significant differences between $\mathrm{AD}$ and $\mathrm{CN}$ subjects. All input features were normalized for the training and testing of the classifiers. Ten input features for the ANN were normalized from -0.9 to 0.9 , because the hyperbolic tangent (tanh) func- tion was used as a neuron output function. The ANN with ten inputs, four hidden layers and one output was trained based on a Levenberg-Marquardt algorithm, in which the learning coefficient was empirically set as 0.9 , a convergence criterion was empirically set as 0.0001 and the maximum number of iterations was set as 200 . Regarding the SVM, input features were normalized from -1.0 to +1.0 . We constructed an SVM classifier with a Gaussian kernel by using the open source software package SVM light [29], which was empirically set as 3.0 for this study. The regularization parameter $\mathrm{C}$ of a cost function for determination of an optimal hyperplane, which can efficiently distinguish between $\mathrm{AD}$ cases and CN subjects, was empirically determined as 180 . The maximum number of iterations was set as 100,000.

\subsubsection{Evaluation of Our Proposed Classification System for AD}

The performance of our proposed method was evaluated based on a receiver operating characteristic (ROC) analysis, where the area under the ROC curve (AUC) was used as a measure of the performance for classification of AD. The ANN and the SVM were trained and tested using a leave-one-out-by-case method. The ROCKIT program was used for creating the ROC curve [30]. The performances of classification of $\mathrm{AD}$ patients based on an ANN and a SVM were compared with each other. In addition, the performances using the morphological and/ or functional image features were compared with those using one of two kinds of image features to investigate the effect of the image features.

The statistical differences in CBFs and cortical thicknesses between $\mathrm{AD}$ patients and $\mathrm{CN}$ subjects in each lobe were estimated with the Student paired $t$ test.

\section{RESULTS}

Figures 3(a) and (b) show therelationship between the average CBFs and cortical thicknesses in the frontal lobe and temporal lobe, respectively. The relationships between the $\mathrm{CBF}$ and cortical thicknesses of the $\mathrm{AD}$ patients and $\mathrm{CN}$ subjects in the frontal lobe and the temporal lobe were overlapped in their feature spaces. There were no statistically significant differences between the two groups in the average CBF or cortical thicknesses of frontal lobe. On the other hand, there were statistically significant differences between the AD patients and the $\mathrm{CN}$ subjects in the average CBF of precuneus $(p<0.05)$ and cortical thicknesses of the temporal lobe $(p<0.05)$. Table 1 shows the results of average CBFs and average thicknesses in cortical regions of each lobe. The average CBFs and cortical thicknesses of $\mathrm{AD}$ patients were $29.3 \mathrm{ml} / 100 \mathrm{ml} / \mathrm{min}$ and $3.15 \mathrm{~mm}$, respectively. On the other hand, the average CBFs and cortical thicknesses of $\mathrm{CN}$ subjects were $33.1 \mathrm{ml} / 100$ 


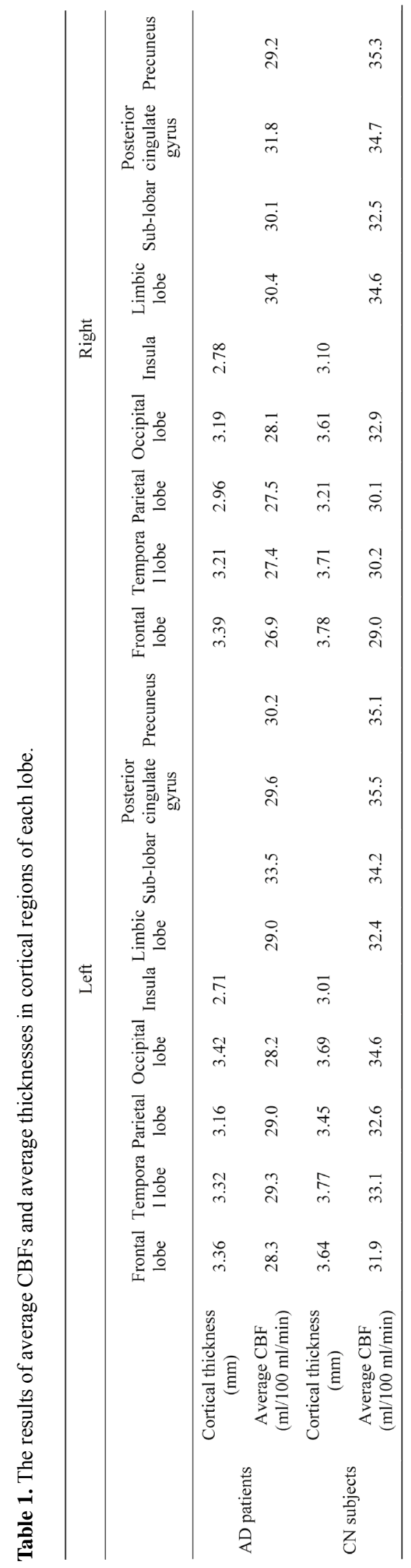




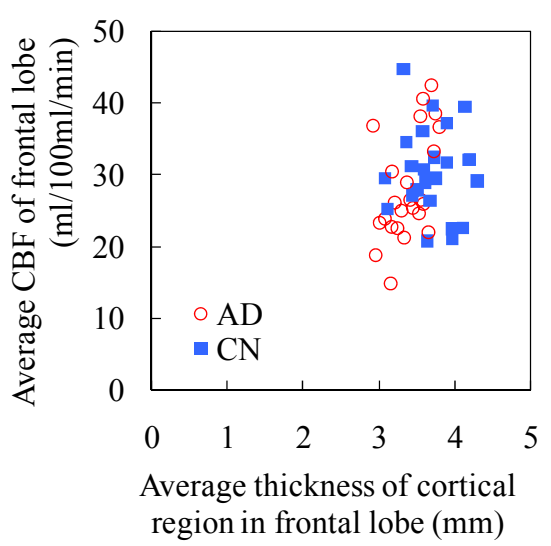

(a)

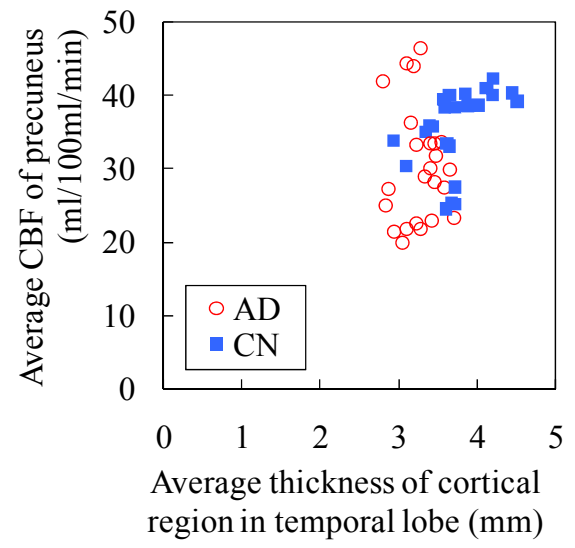

(b)

Figure 3. Relationship between the average $\mathrm{CBF}$ and cortical thicknesses in the frontal lobe (a) and temporal lobe (b). There were no significant differences between the two groups in either the average $\mathrm{CBF}$ or the cortical thicknesses in the frontal lobe (a). On the other hand, there were statistically significant differences between the $\mathrm{AD}$ patients and the $\mathrm{CN}$ subjects in the average $\mathrm{CBF}$ of the precuneus $(\mathrm{p}<0.05)$ and cortical thicknesses of the temporal lobe $(\mathrm{p}<0.05)(\mathrm{b})$.

$\mathrm{ml} / \mathrm{min}$ and $3.50 \mathrm{~mm}$, respectively. In addition, there were statistically significant differences between the AD patients and the $\mathrm{CN}$ subjects in the average CBFs of the left occipital lobe, left posterior cingulate gyrus, left precuneus, and right precuneus and in the cortical thicknesses of the left and right temporal lobe, and left and right insula $(\mathrm{p}<0.05)$.

Figure 4 shows ROC curves for the overall performance of our method in classifying patients with AD and $\mathrm{CN}$ subjects by using the ANN system and the SVM system. The AUC values for the ANN- and the SVMbased systems using both image features were 0.901 and 0.915, respectively. The AUC values for the ANN- and SVM-based systems with the morphological features

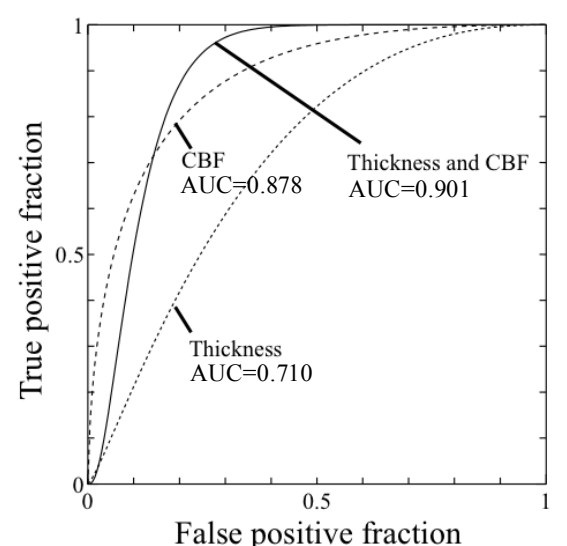

(a)

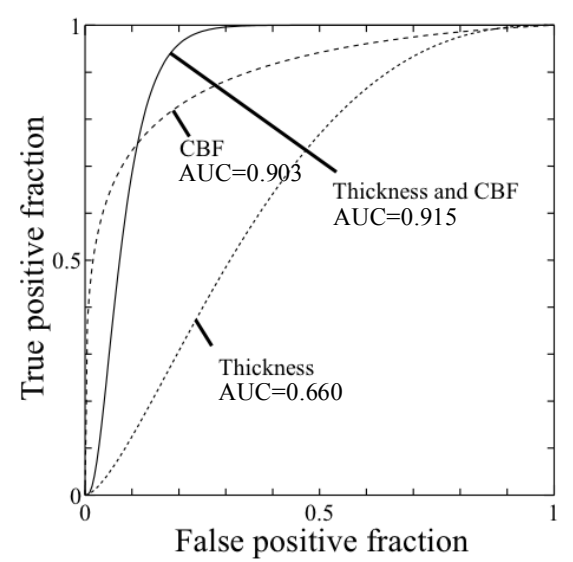

(b)

Figure 4. Receiver-operating characteristic curves for overall performance of our method in classification of patients with $\mathrm{AD}$ and $\mathrm{CN}$ subjects by using the ANN system (a) or the SVM system (b). The areas under the curve in both classifier systems were improved to over 0.9 when calculated using the average $\mathrm{CBFs}$ and thicknesses. The area under the curve in the SVM system was particularly improved, to 0.915 , when the average CBFs and thicknesses were used.

were 0.710 and 0.660 , respectively, and those with the functional features were 0.878 and 0.903 , respectively.

\section{DISCUSSION}

This study showed that the proposed CAD system based on a combination of the morphological and functional image features yielded a higher diagnostic performance for classification of $\mathrm{AD}$ compared with those using only of the two kinds of image features. Although the proposed method misclassified two $\mathrm{AD}$ patients and a $\mathrm{CN}$ subject when using only one type of features, the proposed method correctly identified three cases when both the functional and morphological image features were 
used. Figure 5 shows one (an 81-year-old male with an MMSE score of 20) of the two AD cases that were misclassified using only the cortical thickness features. However, the proposed method correctly classified this $\mathrm{AD}$ case when a combination of the functional and morphological image features was used, which may have been due to thelow CBF value, as shown in Figure 5.

Arimura et al. [17] developed a CAD method for AD with measuring cerebral cortical thicknesses based on normal vectors in 3D T1-weighted MR image. The AUC value in their method was 0.909 in a leave-one-out test method in identification of AD cases among 29 AD cases (mean age: 70; mean MMSE: 20) and $19 \mathrm{CN}$ cases (mean age: 62; mean MMSE: 28). Klöppel et al. [18] proposed a CAD method by using a linear SVM to classify the grey matter segment of T1-weighted MR scans, and tested their method for distinguishing $\mathrm{AD}$ from $\mathrm{CN}$ cases. According to their results, 95\% (a sensitivity of $95.0 \%$ and a specificity of $95.0 \%$ ) of $\mathrm{AD}$ patients were distinguished in a leave-one-out test among 20 AD cases (mean age: 81; mean MMSE: 17) and 20 CN cases (mean age: 80 ; mean MMSE: 29). Colliot et al. [19] reported that their developed method based on hippocampal volumes in 3D T1-weightedMR images achieved a classification rate of $84 \%$ (a sensitivity of $84 \%$, a specificity of $84 \%$, and a AUC value of 0.913 ) between 25 AD patients (mean age: 73; mean MMSE: 24) and 25 controls (mean age: 64; MMSE: no description). Ramirez et al. [20] developed a CAD system for $\mathrm{AD}$ patients based on a baseline principal component analysis (PCA) system in brain SPECT images. They re-

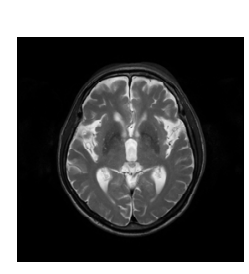

(a)

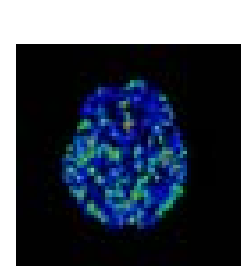

(b)

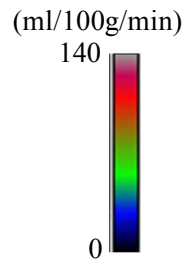

(d)

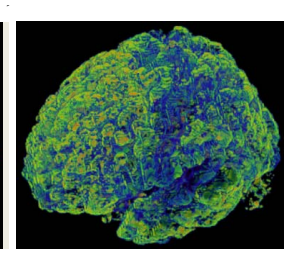

(e) (c)

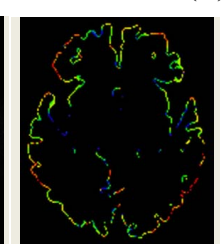

ported a sensitivity of $100 \%$, a specificity of $92.7 \%$, and an accuracy of $96.9 \%$ for $41 \mathrm{AD}$ cases and $56 \mathrm{CN}$ cases (age and MMSE were not mentioned). On the other hand, in our results, the AUC values using the SVM-based system when individually using morphological features and functional features were 0.660 and 0.903 , respectively. In comparison between this study and past studies, the AUC value of the proposed method was lower than conventional methods when using only morphological image features. However, the AUC value achieved 0.915 when applying the combination of two image features.

The proposed CAD system for differential diagnosis of $\mathrm{AD}$ has the advantage that it can provide the functional and morphological image features by means of only an MR examination without contrast medium. If the differential diagnostic accuracy of AD could be improved by using our proposed system, then highly accurate AD diagnosis would be achievable by only an MR examination without contrast medium, and the examination burden for patients would be mitigated.

Our proposed method has three limitations. The first limitation is that the classification results were affected bysome artifacts on MR imaging. Such artifacts were particularly prevalent when using the ASL technique, and included motion artifacts, N/2 ghost artifacts, which area type of magnetic susceptibility artifacts, and the artifacts caused by blood flow in the vessels. Figure 6 shows an AD case (a 72-year-old man with an MMSE score of 23) that was incorrectly classified as a CN subject due to overestimation of the $\mathrm{CBF}$ value by motion artifact. The second limitation was the number of cases

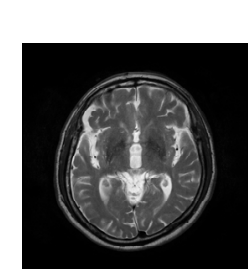

(a)

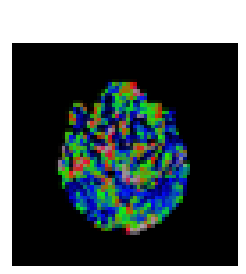

(b)

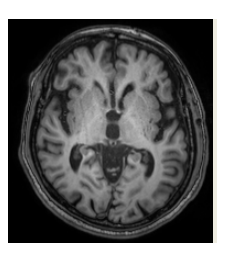

(c)

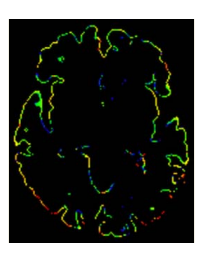

(d)

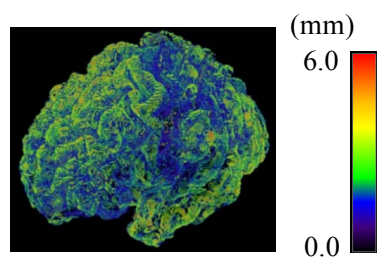

(e)
Figure 5. Original MR images and color-coded maps of cerebral cortical thicknesses in patients with Alzheimer's disease (age: 81 years; gender: male; mini-mental statement examination score: 20): (a) an original T2-weighted image; (b) an original CBF map image obtained by the ASL technique; (c) an original T1-weighted image; (d) a color-coded axial map of cortical thicknesses; (e) a color-coded volume-rendering map of cortical thicknesses.
Figure 6. Original MR images and color-coded maps of cerebral cortical thicknesses in patients with Alzheimer's disease (age: 72 years; gender: male; mini-mental statement examination score: 23): (a) an original T2-weighted image; (b) an original CBF map image obtained by the ASL technique; (c) an original T1-weighted image; (d) a color-coded axial map of cortical thicknesses; (e) a color-coded volume-rendering map of cortical thicknesses. 
used to train the classifier. Both machine learning classifiers, SVM and ANN, were trained with $15 \mathrm{AD}$ cases and $15 \mathrm{CN}$ subjects in our proposed method. It will be necessary to collect more data sets in order to improve the classification accuracy, because the number of training cases greatly influences the result [31]. The third limitation is that additional classifiers will need to be tested, because only the SVM and ANN were evaluated in this study.

\section{CONCLUSION}

We have developed a computer-aided classification system for $\mathrm{AD}$ patients based on a combination of morphological and functional image features obtained by MR imaging without contrast medium. Our preliminary results suggest that the proposed method may have feasibility for the classification of AD patients by using morphological and functional image features.

\section{ACKNOWLEDGEMENTS}

The authors are grateful to all members of the Arimura-Laboratory (http://www.shs.kyushu-u.ac.jp/ arimura) for their valuable comments and helpful discussion. The authors thank the members of the Division of Radiology of the Department of Medical Technology at Kyushu University Hospital. This work was supported by JSPS KAKENHI Grant Number 21791199.

\section{REFERENCES}

[1] World Health Organization (2006) The World Health Report 2006, Working Together for Health. http://www.who.int/whr/2006/en/index.html

[2] Chris, H., Vikas, S., Guofan, X. and Sterling C.J. (2011) The Alzheimer's disease neuroimaging initiative, predictive markers for $\mathrm{AD}$ in a multi-modality framework: An analysis of MCI progression in the ADNI population. NeuroImage, 55, 574-589. http://dx.doi.org/10.1016/j.neuroimage.2010.10.081

[3] Catriona, D.M., David, C., Stephen P.M. and A. Peter P. (2001) Risk factors for dementia. Advances in Psychiatric Treatment, 7, 24-31. http://dx.doi.org/10.1192/apt.7.1.24

[4] Yoshitake, T., Kiyohara, Y., Kato, I., Ohmura, T., Iwamoto, H., Nakayama, K., Ohmori, S., Nomiyama, K., Kawano, H., Ueda, K., Sueishi, K., Tsuneyoshi, M. and Fujishima, M. (1995) Incidence and risk factors of vascular dementia and Alzheimer's disease in a defined elderly Japanese population. Neurology, 45, 1161-1168. http://dx.doi.org/10.1212/WNL.45.6.1161

[5] Claire, M. and Christian, D. (2006) Alzheimer disease: Progress or profit? Nature Medicine, 12, 780-784. http://dx.doi.org/10.1038/nm0706-780

[6] Bronge, L., Bogdanovic, N. and Wahlund, L.O. (2002) Postmortem MRI and histopathology of white matter changes in Alzheimer brains: A quantitative, comparative study. Dementia and Geriatric Cognitive Disorders, 13,

\section{5-212. http://dx.doi.org/ 10.1159/000057698}

[7] Brun, A. and Englund, E. (1986) A white matter disorder in dementia of the Alzheimer type: A pathoanatomical study. Annals of Neurology, 19, 253-262.

http://dx.doi.org/10.1002/ana.410190306

[8] Englund, E. (1998) Neuropathology of white matter changes in Alzheimer's disease and vascular dementia. Dementia and Geriatric Cognitive Disorders, 9, 6-12. http://dx.doi.org/10.1159/000051183

[9] Agosta, F., Pievani, M., Sala, S., Geroldi, C., Galluzzi, S., Frisoni, G.B. and Filippi, M., (2011) White matter damage in Alzheimer disease and its relationship to gray matter atrophy. Radiology, 258, 853-863.

http://dx.doi.org/10.1148/radiol.10101284/-/DC1

[10] Matsuda, H. (2007) Cerebral blood flow and metabolic abnormalities in Alzheimer's disease. Annals of Nuclear Medicine, 15, 85-92. http://dx.doi.org/10.1007/BF02988596

[11] Hirata, Y., Matsuda, H. and Nemoto, K. (2005) Voxelbased morphometry to discriminate early Alzheimer's disease from controls. Neuroscience Letter, 382, 269-274. http://dx.doi.org/10.1016/j.neulet.2005.03.038

[12] Matsuda, H. (2007) Role of neuroimaging in Alzheimer's disease, with emphasis on brain perfusion SPECT. Journal of the Nuclear Medicine, 48, 1289-1300. http://dx.doi.org/10.2967/jnumed.106.037218

[13] Li, S., Shi, F., Pu, F., Li, X., Jiang, T., Xie, S. and Wang, Y. (2007) Hippocampal shape analysis of Alzheimer disease based on machine learning methods. American Journal of Neuroradiology, 28, 1339-1345. http://dx.doi.org/10.3174/ajnr.A0620

[14] Petersen, E.T., Zimine, I., Ho, Y.C. and Golay, X. (2006) Non-invasive measurement of perfusion: A critical review of arterial spin labelling techniques. British Journal of Radiology, 79, 688-701. http://dx.doi.org/10.1259/bjr/67705974

[15] Kim, S.G. and Tsekos, N.V. (1997) Perfusion imaging by a flow-sensitive alternating inversion recovery (FAIR) technique: Application to functional brain imaging. Magnetic Resonance in Medicine, 37, 425-35. http://dx.doi.org/10.1002/mrm.1910370321

[16] Yoshiura, T., Hiwatashi, A., Noguchi, T., Yamashita, K., Ohyagi, Y., Monji, A., Nagao, E., Kamano, H., Togao, O. and Honda, H. (2009) Arterial spin labelling at 3-T MR imaging for detection of individuals with Alzheimer's disease. European Radiology, 19, 2819-2825. http://dx.doi.org/10.1007/s00330-009-1511-6

[17] Arimura, H., Yoshiura, T., Kumazawa, S., Tanaka, K., Koga, H., Mihara, F., Honda, H., Sakai, S., Toyofuku, F. and Higashida, Y. (2008) Automated method for identification of patients with Alzheimer's disease based on three-dimensional MR images. Academic Radiology, 15, 274-284. http://dx.doi.org/10.1016/j.acra.2007.10.020

[18] Klöppel, S., Stonnington, C.M., Chu, C., Draganski, B., Scahill, R.I., Rohrer, J.D., Fox, N.C., Jack, C.R., Ashburner, Jr, J. and Frackowiak, R.S.J. (2008) Automatic classification of MR scans in Alzheimer's disease. Brain, 131, 681-689. http://dx.doi.org/10.1093/brain/awm319 
[19] Colliot, O., Chételat, G., Chupin, M., Desgranges, B., Magnin, B., Benali, H., Dubois, B., Garnero, L., Eustache, F. and Lehéricy, S. (2008) Discrimination between Alzheimer disease, mild cognitive impairment, and normal aging by using automated segmentation of the hippocampus. Radiology, 248, 194-201. http://dx.doi.org/10.1148/radiol.2481070876

[20] Ramírez, J., Górriz, J.M., Segovia, F., Chaves, R., SalasGonzalez, D., López, M., Álvarez, I. and Padilla, P. (2010) Computer aided diagnosis system for the Alzheimer's disease based on partial least squares and random forest SPECT image classification. Neuroscience Letters, 472, 99-103. http://dx.doi.org/10.1016/j.neulet.2010.01.056

[21] Tokunaga, C., Arimura, H., Yoshiura, T., Ohara, T., Yamashita, Y., Kobayashi, K., Magome, T., Nakamura, Y., Honda, H., Hirata, H., Ohki, M. and Toyofuku, F. (2013) Automated measurement of three-dimensional cerebral cortical thickness in Alzheimer's patients using localized gradient vector trajectory in fuzzy membership maps. Journal of Biomedical Science and Engineering, 6, 327336. http://dx.doi.org/10.4236/jbise.2013.63A042

[22] Petersen, E.T., Lim, T. and Golay, X. (2006) Model-free arterial spin labeling quantification approach for perfusion MRI. Magnetic Resonance in Medicine, 55, 219-232. http://dx.doi.org/10.1002/mrm.20784

[23] Talairach, J. and Tournoux, P. (1988) Co-planar stereotaxic atlas of the human brain: 3-Dimensional proportional system: An approach to cerebral imaging. Thieme Medical Publishers, Inc., New York.

[24] Lancaster, J.L., Woldorff, M.G., Parsons, L.M., Liotti, M., Freitas, C.S., Rainey, L., Kochunov, P.V., Nickerson, D., Mikiten, S.A. and Fox, P.T. (2000) Automated Talairach Atlas labels for functional brain mapping. Human Brain Mapping, 1, 120-131.

http://dx.doi.org/10.1002/1097-0193(200007)10:3<120:: AID-HBM30>3.0.CO;2-8
[25] Lancaster, J.L., Rainey, L.H., Summerlin, J.L., Freitas, C.S., Fox, P.T., Evans, A.C., Toga, A.W. and Mazziotta J.C. (1997) Automated labeling of the human brain: A preliminary report on the development and evaluation of a forward-transform method. Human Brain Mapping, 5, 238-242.

http://dx.doi.org/10.1002/(SICI)1097-0193(1997)5:4<238 $\because$ AID-HBM6>3.0.CO;2-4

[26] Lee, S., Wolberg, G. and Shin, S.Y. (1997) Scattered data interpolation with multilevel B-splines. IEEE Transactions on Visualization and Computer Graphics, 3, 228244. http://dx.doi.org/10.1109/2945.620490

[27] Laboratory of Neuro Imaging (2012) International consortium for brain mapping. http://www.loni.ucla.edu/ICBM/

[28] Alsop, D.C., Detre, J.A. and Grossman, M. (2000) Assessment of cerebral blood flow in Alzheimer's disease by spinlabeled magnetic resonance imaging. Annals of Neurology, 47, 93-100. http://dx.doi.org/10.1002/1531-8249(200001)47:1<93::AI D-ANA15>3.0.CO;2-8

[29] Joachims, T. (2008) SVMlight, Cornell University. http://svmlight.joachims.org/

[30] Metz, C.E., Herman, B.A. and Roe, C.A. (1998) Statistical comparison of two ROC curve estimates obtained from partially-paired datasets. Medical Decision Making, 18, 110-121. http://dx.doi.org/10.1177/0272989X9801800118

[31] Wua, T.K., Huangb, S.C. and Mengc, Y.R. (2008) Evaluation of ANN and SVM classifiers as predictors to the diagnosis of students with learning disabilities. Expert Systems with Applications, 34, 1846-1856. http://dx.doi.org/10.1016/j.eswa.2007.02.026 